\title{
Hadroproduction of $t$ anti-t pair in association with an isolated photon at NLO accuracy matched with parton shower
}

\author{
Adam Kardos ${ }^{a}$ and Zoltán Trócsányi ${ }^{b}$ \\ ${ }^{a}$ MTA-DE Particle Physics Research Group, \\ University of Debrecen, H-4010 Debrecen P.O.Box 105, Hungary \\ ${ }^{b}$ Institute of Physics and MTA-DE Particle Physics Research Group, \\ University of Debrecen, H-4010 Debrecen P.O. Box 105, Hungary \\ E-mail: kardos.adam@science.unideb.hu, zoltan.trocsanyi@cern.ch
}

\begin{abstract}
We simulate the hadroproduction of a t $\overline{\mathrm{t}}$-pair in association with a hard photon at LHC using the PowHel package. These events are almost fully inclusive with respect to the photon, allowing for any physically relevant isolation of the photon. We use the generated events, stored according to the Les-Houches event format, to make predictions for differential distributions formally at the next-to-leading order (NLO) accuracy and we compare these to existing predictions accurate at NLO using the smooth isolation prescription of Frixione. Our fixed-order predictions include the direct-photon contribution only. We also make predictions for distributions after full parton shower and hadronization using the standard experimental cone-isolation of the photon.
\end{abstract}

KeYwords: Monte Carlo Simulations, NLO Computations

ARXIV EPRINT: 1406.2324 


\section{Contents}

1 Introduction 1

2 Details of the implementation 3

3 NLO-LHE comparison $\quad 5$

$\begin{array}{lll}4 & \text { Photon isolation as a generation cut } & 7\end{array}$

5 Independence of the generation isolation $\quad 9$

6 Estimation of the non-pertrubative contribution 10

$\begin{array}{lll}7 & \text { Effect of the parton shower } & 13\end{array}$

8 Predictions 13

9 Conclusions $\quad 16$

\section{Introduction}

Isolated hard photons are important experimental tools for a variety of processes at the LHC. Most notably, one of the cleanest channels to identify the Standard Model (SM) Higgs particle is its decay into a pair of hard photons. Although this channel has a small (about $0.2 \%$ ) branching ratio as compared to the hadronic and leptonic channels, the spectacular resolution of the electromagnetic calorimeters of the ATLAS and CMS detectors and the relatively low background made this as one of the prime discovery channels $[1,2]$.

From the theoretical point of view isolated hard photons are rather cumbersome objects. Unlike leptons, the photons couple directly to quarks. If the quark that emits the photon is a light quark, treated massless in perturbative QCD, then the emission is enhanced at small angles and in fact, becomes singular for strictly collinear emission. The usual experimental definition of an isolated photon allows for small hadronic activity even inside the isolation cone. Due to the divergence of the collinear emission, this isolation cannot be implemented directly in a perturbative computation at leading-order (LO) accuracy because even small hadronic activity inside the cone leads to infinite results.

Of course, one can approximate the experimental definition with complete isolation of the photon from the coloured particles inside a fixed cone and obtain a perturbative prediction at LO. The problem however, comes back with a different face if we want to define the isolated photon in a computation at the next-to-leading order (NLO) accuracy. At NLO there are two kinds of radiative corrections: (i) the virtual one with the same 
final state as the Born contribution, but including a loop and (ii) the real one that involves the emission of a real parton in the final state. These two contributions are separately divergent, but their sum is finite for infrared (IR) safe observables according to the KLN theorem $[3,4]$. The IR-safe observables are represented by a jet function $J_{m}$, where $m$ is the number of partons in the final state: for an $n$-jet measure $m=n$ at LO and for the virtual corrections, while $m=n+1$ in the real correction.

There exist general methods (see e.g. ref. [5]) to combine the real and virtual corrections for infrared (IR) safe observables $J_{m}$, for which $J_{n+1}$ tends to $J_{n}$ smoothly in kinematically degenerate regions of the phase space, namely when two final-state partons become collinear or a final-state gluon becomes soft. The problem with the isolated-photon cross section in perturbative QCD is that the cone-photon isolation is not IR safe beyond LO. The extra gluon in the real radiation may be radiated within the isolation cone in which case the event will be cut even if the gluon energy tends to zero.

There are ways to make predictions for photon production in perturbation theory, but all have drawbacks. In a pioneering work [6] the measurement of the inclusive photon cross section was advocated, but that is not very useful from the experimental point of view. In ref. [7] an isolation procedure was proposed that is similar in spirit to the case of inclusive cross section, yet provides a smooth isolation prescription that is IR safe at all orders in perturbation theory. However, the implementation of the smooth prescription experimentally is very cumbersome as it requires very fine granularity of the detector, so it has never become popular among experimenters.

There is a precise way of defining the isolated photon theoretically, but that requires the inclusion of the photon fragmentation component as well (see e.g. [8]). The drawback of this approach is the need for non-perturbative input and the extra computational effort for a contribution that is mostly discarded when the experimental isolation is used (cone with small hadronic activity inside that is described by the fragmentation contribution). Thus one would be tempted to neglect the fragmentation contribution, which is however, uncontrolled from the theoretical point of view and thus is not a viable option in a fixedorder computation.

In the last decade new approaches were proposed to make predictions that are formally accurate to NLO but include the advantage of event simulations of the shower Monte Carlo (SMC) programs [9-11]. By now many processes have been included in the generic frameworks of these NLO+PS approaches, the aMCatNLO [12] and the POWHEG-BOX [13] codes. In a series of papers we combined the POWHEG-BOX with the HELAC-NLO package [14] into PowHel to make predictions for the hadroproduction of a $\mathrm{t} \overline{\mathrm{t}}$-pair in association with a hard boson (scalar [15], pseudoscalar [16], vector [17] or jet [18]). The only missing boson of the SM in this list is the hard photon. In view of the above, the reason is clear: the photon has to be isolated, which makes this computation more involved than for the other cases.

In this paper we use the PowHel framework to simulate events containing direct photons only, that is we neglect the fragmentation contribution. We generate the events with loose isolation cut, resulting in an almost inclusive event sample. We argue that with sufficiently loose generation isolation the fragmentation contribution should be indeed small. The output of the POWHEG-BOX consists of pre-showered events stored according to the Les 
Houches accord (LHEs) [19]. The LHEs when fed into a SMC, result in showered events on which the usual experimental cone isolation can be applied. We discuss the validity of this approach on the example of $W \gamma$ hadroproduction for which predictions including a modelling of fragmentation as well as experimental results exist. Using events generated with loose isolation cuts, we make predictions to $t \overline{\mathrm{t}} \gamma$ hadroproduction, but the approach is general and can be used to make predictions for any other process that involves isolated hard photons in the final state at NLO accuracy matched with PS.

\section{Details of the implementation}

PowHel is a computational framework composed of the POWHEG-BOX [13] and the HELAC-NLO [14] packages to provide predictions at the hadron level with NLO QCD accuracy in the hard process. The essential ingredients needed for a particular process are the matrix elements for the Born, virtual and real-emission contributions, spin- and colourcorrelated matrix elements and a suitable phase space for the Born process.

The matrix elements are provided by HELAC-NLO while the Born phase space is constructed by us using the relatively simple kinematics at the Born level. The Born phase space is generated with the help of one kinematic invariant and three angles. An overall azimuth is kept fixed and randomly reinstated at the end of the calculation as a common practice in POWHEG-BOX. Matrix elements are generated for the following subprocesses: $\mathrm{q} \overline{\mathrm{q}} \rightarrow \gamma \mathrm{t} \overline{\mathrm{t}}, g g \rightarrow \gamma \mathrm{t} \overline{\mathrm{t}}$ (tree-level for the Born process and at one-loop for the virtual) and $\mathrm{q} \overline{\mathrm{q}} \rightarrow \gamma \mathrm{t} \overline{\mathrm{t}} g, g g \rightarrow \gamma \mathrm{t} \overline{\mathrm{t}} g$ for the real emission $(\mathrm{q} \in\{\mathrm{u}, \mathrm{d}, \mathrm{c}, \mathrm{s}, \mathrm{b}\})$. The ordering among particles follows the convention of POWHEG-BOX: non-QCD particles, massive quarks, massless partons. Matrix elements for all other subprocesses are obtained from these by means of crossing.

All matrix elements, including the crossed ones, are compared to the stand-alone version of HELAC-NLO in several, randomly chosen phase-space points. The internal consistency between the Born, spin-, colour-correlated and real-emission matrix elements is checked by comparing the limit of the real-emission part and the corresponding counterterms in all kinematically degenerate regions of the phase space.

In order to check the whole implementation we compare differential distributions to those in ref. [20] using the LHC setup in the published paper: the calculation was performed for LHC at centre-of-mass energy $\sqrt{s}=14 \mathrm{TeV}$ with a CTEQ6L1 and CTEQ6.6M PDF at LO and NLO accuracy and a one- and two-loop running $\alpha_{s}$, respectively. The mass of the t-quark was $m_{\mathrm{t}}=172 \mathrm{GeV}$, the fine-structure constant, was set to $\alpha_{\mathrm{EM}}=1 / 137$. The renormalization and factorization scales were set fixed, equal to $m_{\mathrm{t}}$. In the analysis a photon was required to be hard, $p_{\perp, \gamma}>20 \mathrm{GeV}$ and the smooth isolation of Frixione [7] was employed with isolation parameters $\delta_{0}=0.4$ and $\epsilon_{\gamma}=n=1$. The cross sections obtained with PowHel are enlisted on table 1 . We found complete agreement with the predictions of [20] both for the cross sections and for the available distributions as well. Two out of these are depicted in figure 1.

Having checked the implementation of the NLO computation, we generated events with the POWHEG-BOX. The final state in the Born contribution, $t \bar{t} \gamma$, is composed of two 


\begin{tabular}{|c|c|c|}
\hline$\mu$ & $\sigma_{\mathrm{LO}}^{\mathrm{PH}}[\mathrm{pb}]$ & $\sigma_{\mathrm{NLO}}^{\mathrm{PH}}[\mathrm{pb}]$ \\
\hline $2 m_{\mathrm{t}}$ & $1.519 \pm 0.004$ & $2.58 \pm 0.03$ \\
\hline$m_{\mathrm{t}}$ & $1.968 \pm 0.005$ & $2.94 \pm 0.04$ \\
\hline$m_{\mathrm{t}} / 2$ & $2.614 \pm 0.006$ & $3.33 \pm 0.06$ \\
\hline
\end{tabular}

Table 1. Cross sections obtained with PowHel at LO and NLO accuracy using the setup and cuts of [20]. The renormalization and factorization scales are made equal to $\mu$.
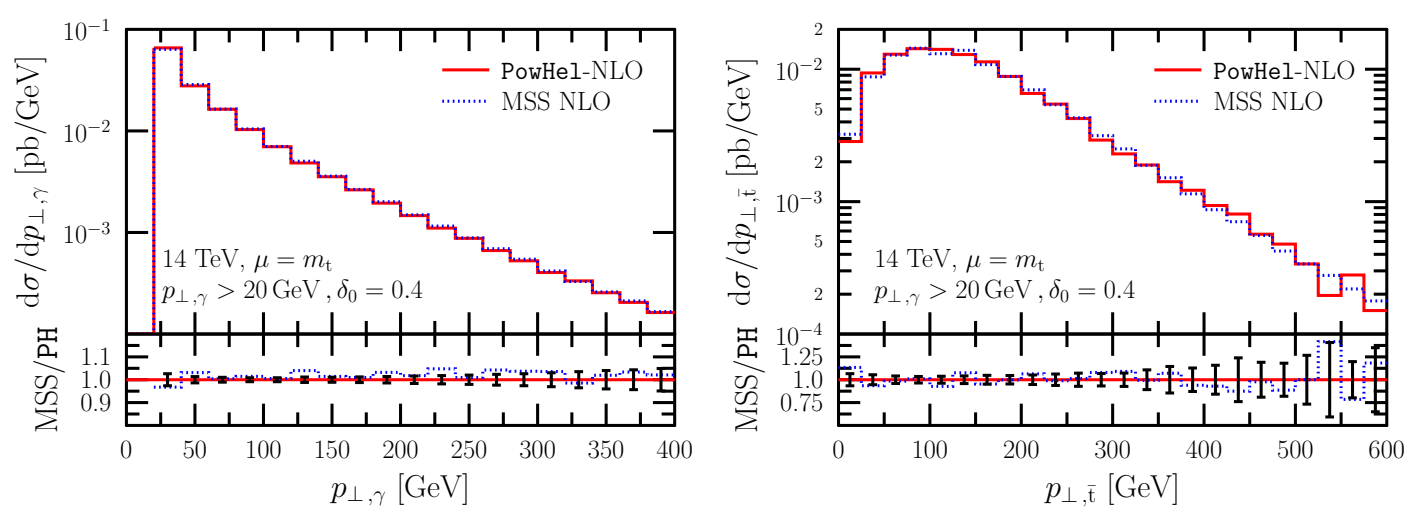

Figure 1. Comparison between PowHel and [20] at the central scale with NLO accuracy for the differential cross section as a function of the transverse momentum of the photon and anti-t quark. Lower panels depict the ratio of predictions in [20] (MSS) to ours. The uncertainties appearing on the lower panels only take into account the statistical uncertainty of our calculation.

massive and one massless particles. The cross section when the photon is emitted from a massless (anti)quark can become singular. This can happen when the photon is emitted by one massless (anti)quark from the initial state, or from a final state one in the real-emission contribution. These configurations have to be avoided such that the physical cross sections for isolated photon production do not depend on the actual implementation.

Let us first focus only on the singular radiation present at the Born level. In this case there are two simple solutions to avoid infinite contributions to the cross section. The first is a generation cut [18], which if applied on the transverse momentum of the photon, can avoid the singularity. This cut has to be sufficiently small so that when physical cuts are applied, the prediction becomes independent of this generation cut. Although this method offers an easy way to avoid the singularity, yet we end up generating events mostly with photons having small transverse momentum. Hence the majority of events will be generated in a region of phase space which has no physical importance and the efficiency of the event generation is small.

The other solution is the inclusion of a suppression factor [21] which can be used to enhance event generation in certain regions of the phase space. The distributions are always independent of the suppression used as events are generated according to a cross section obtained with a suppression factor, but the weight of each event is multiplied by the inverse of the suppression factor (for details see [22]). In our calculation the analytical 
form of suppression was chosen to be

$$
\mathcal{F}_{\text {supp }}=\frac{1}{1+\left(\frac{p_{\perp, \text { supp }}^{2}}{p_{\perp, \gamma}^{2}}\right)^{i}},
$$

and we found $i=2$ a suitable choice and $p_{\perp \text {,supp }}=100 \mathrm{GeV}$ was set throughout the whole calculation. It is not necessary, yet we included also the generation cut on the transverse momentum of the photon, by requiring the transverse momentum of the photon in the underlying phase space to be larger than $15 \mathrm{GeV}$. We checked that this cut does not affect our predictions with physical cuts larger than $15 \mathrm{GeV}$. Our strategy to handle singularities coming from collinear photon-emission from final state massless (anti)quarks will be covered section 4.

In order to speed up the event generation the real emission part can be decomposed into a singular and finite contribution such that the former contains all the kinematically degenerate regions of phase space, while the latter is finite over the whole phase space. When this decomposition is implemented the POWHEG Sudakov factor is evaluated with only the singular contribution. For the decomposition we used the original suggestion of [13] which became standard in all calculations done with the help of POWHEG-BOX. Beside of this decomposition and the generation isolation nothing is taken into account which could alter the shape of the POWHEG Sudakov, that is the matching systematics. In particular, we have not used the hfact option which is only used in [23] and in all the other cases the separation of real emission contribution, mentioned above, was considered only.

\section{NLO-LHE comparison}

In this and all the upcoming sections predictions are made for proton-proton collisions at $\sqrt{s}=8 \mathrm{TeV}$ with the following parameters: CT10nlo PDF using LHAPDF [24] with a 2-loop running $\alpha_{s}$ considering 5 massless quark-flavours, $m_{\mathrm{t}}=172.5 \mathrm{GeV}, \alpha_{\mathrm{EM}}=1 / 137$. For our default scale we decided to use a dynamical one, the half of the sum of transverse masses of all final-state particles:

$$
\mu_{0}=\frac{1}{2} \hat{H}_{\perp}=\frac{1}{2}\left(m_{\perp, \mathrm{t}}+m_{\perp, \overline{\mathrm{t}}}+p_{\perp, \gamma}\right),
$$

where the hat reminds us that underlying-Born kinematics was used to evaluate the sum. For the NLO-LHE comparison the following set of cuts was employed:

- The photon had to be hard enough, $p_{\perp, \gamma}>30 \mathrm{GeV}$.

- The photon was constrained into the central region, $\left|y_{\gamma}\right|<2.5$.

- To avoid the quark-photon singularity a Frixione-isolation was used with $\delta_{0}=0.4$ and $\epsilon_{\gamma}=n=1$.

The cross section at LO and NLO accuracy as a function of the equal renormalization and factorization scale normalized to the default scale $\mu_{0}$ is shown in figure 2 . We find 


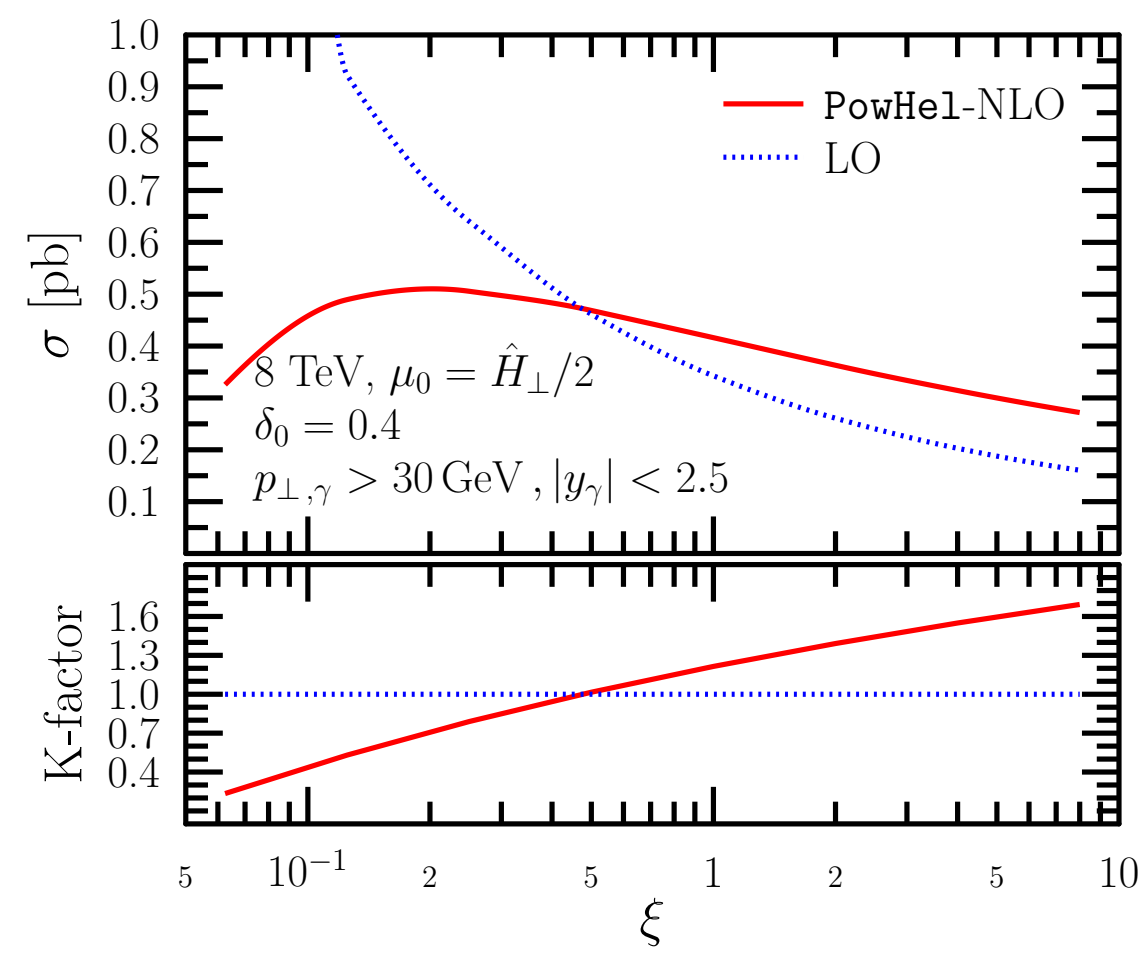

Figure 2. Cross section with cuts listed in the text and also shown in the figure at LO (blue dotted) and at NLO (red solid) accuracy as a function of the equal renormalization and factorization scale normalized to the default scale $\mu_{0}$. The lower panel shows the NLO K-factor.
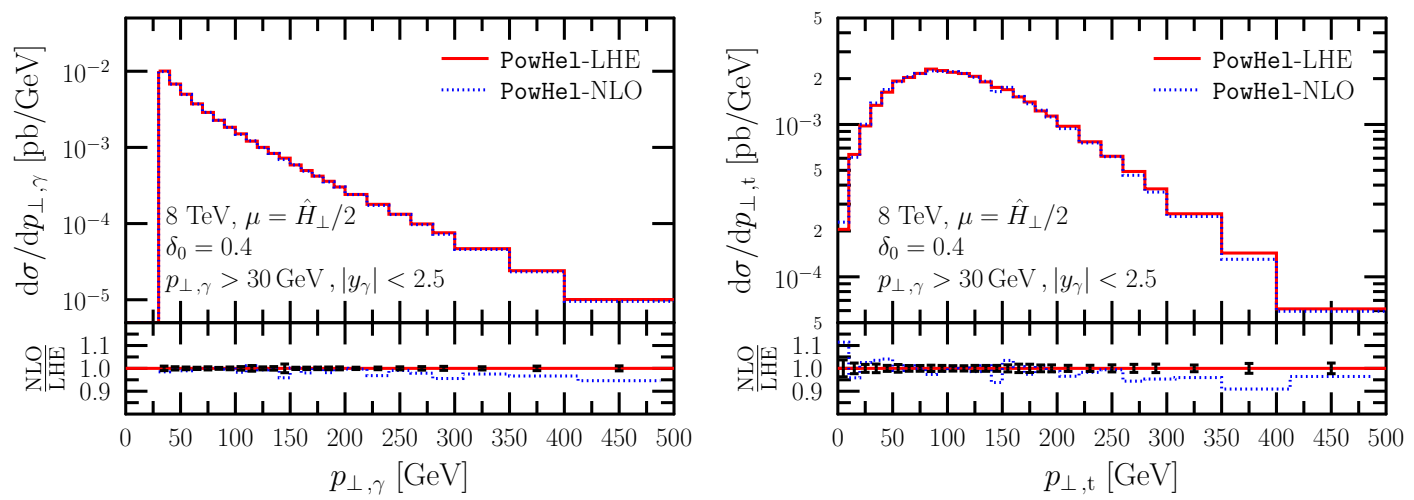

Figure 3. Comparison between predictions from LHEs (solid red) and at NLO (blue dashed) using Frixione isolation for the transverse momentum of the photon and t-quark. On the lower panel the ratio of the two predictions is shown.

significant reduction of the scale dependence and an NLO K-factor $K=1.21$ at our default scale choice.

Next we turn to comparisons of predictions at NLO accuracy with those obtained from the pre-showered events. With this comparison our only aim is to demonstrate that our framework can generate meaningful pre-showered events using the Frixione isolation (the standard in fixed-order calculations). On figures 3-5 six sample distributions are depicted 

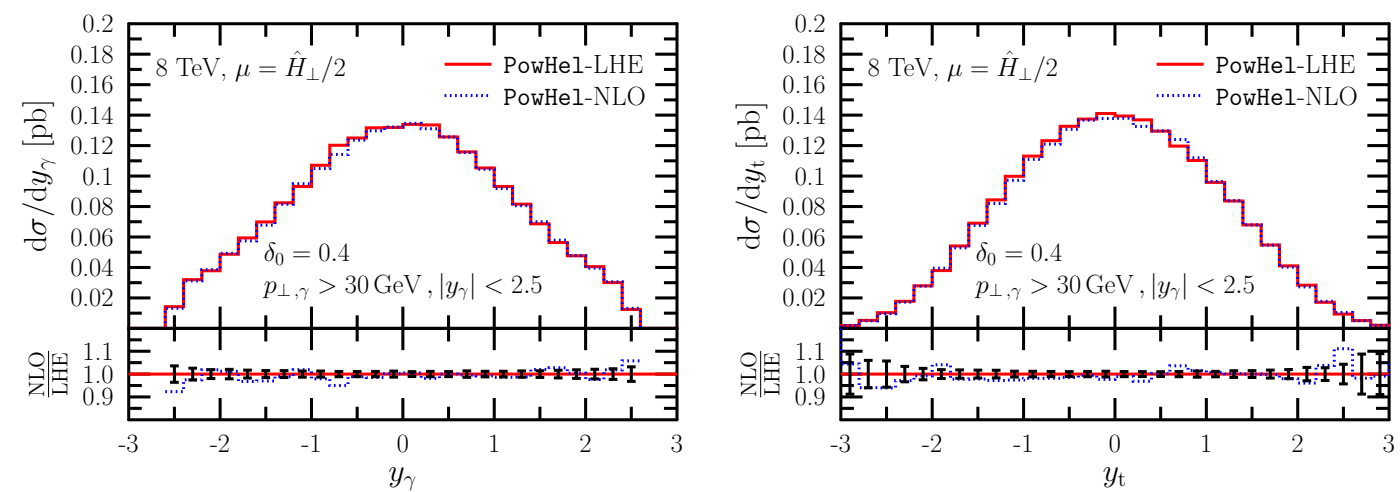

Figure 4. The same as figure 3 for the rapidities of the photon and the t-quark.
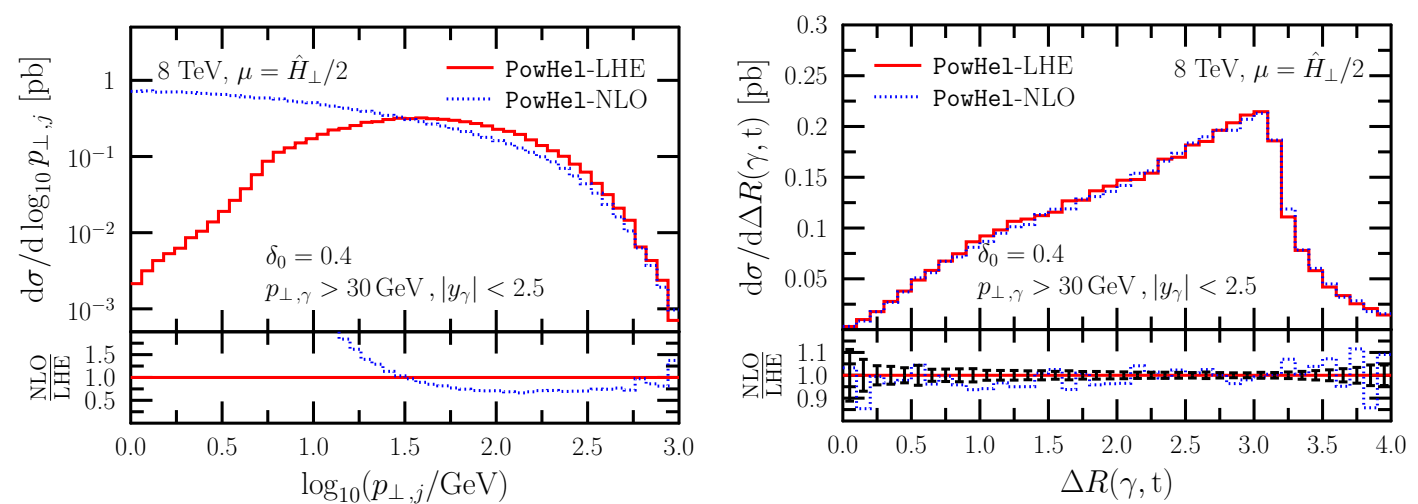

Figure 5. The same as figure 3 but the differential cross section is depicted as a function of the logarithm of the extra-parton transverse momentum and the separation of the photon and the t-quark. The separation is defined in the rapidity-azimuthal angle plane.

to illustrate the effect of the POWHEG Sudakov factor. In general we find agreement between the corresponding predictions except for the transverse-momentum distribution for the extra parton (left plot of figure 5). The effect of the POWHEG Sudakov suppression is clearly visible in the low $p_{\perp}$ region where the radiation activity is highly limited, as expected. The presence of the extra cut in the real-emission part (the Frixione isolation) causes small distortion in the Sudakov shape as seen at about 0.75. in the left plot of figure 5. These comparisons show good agreement between the fixed-order predictions and those from the pre-showered events. The visible differences can be accounted for the effect of the POWHEG Sudakov factor. It is worth mentioning that the formal accuracy is still NLO, the difference is due to higher order terms.

\section{Photon isolation as a generation cut}

When photons are produced with massless partons in the final state the usual soft/collinear divergences coming from parton-parton splittings are accompanied by a new type of collinear splitting, namely the quark-photon one. The singularity produced by a collinear photon emission off a massless (anti)quark can be absorbed into the photon fragmentation 
function, decomposing the cross section into direct photon production and a fragmentation contribution.

The only known solution that leads to an IR-safe cross section at all orders in perturbation theory that avoids the fragmentation contribution is offered in ref. [7] where QCD activity is considered in a continuously shrinking cone around the photon such that the allowed activity decreases with decreasing cone size.

While in a theoretical calculation the shrinking cone size can be easily implemented, in an experiment the finite resolution of the detector does not allow for taking the smooth limit. As a result most of the experiments adopt a different isolation criterion: reduced hadronic activity is allowed around the photon in a cone with finite size such that for the total hadronic transverse energy inside the cone

$$
E_{\perp, \text { had }}=\sum_{i \in \text { tracks }} E_{\perp, i} \Theta\left(R_{\gamma}-R\left(p_{\gamma}, p_{i}\right)\right)<E_{\perp, \text { had }}^{\max } .
$$

In eq. (4.1) $E_{\perp, i}$ is the transverse energy of the $i$ th track, $R_{\gamma}$ is the isolation cone size, $R\left(p_{\gamma}, p_{i}\right)$ is the separation between the photon and the $i$ th track measured in rapidityazimuthal angle plane, while $E_{\text {had }}^{\max }$ is the maximal hadronic energy allowed to be deposited in the cone of $R_{\gamma}$ around the photon. In the following we call this quantity hadronic or partonic leakage depending on whether the process is considered on the hadron or the parton level. In a fixed-order calculation an isolation of the form of eq. (4.1) does not completely remove the singularity of collinear quark-photon emission and therefore, cannot be applied. Setting $E_{\perp \text {,had }}^{\max }=0$ removes this singularity, but cuts into the phase space of soft gluon emission in the real correction, hence it is not IR-safe.

As shown in the previous section, there is one photon isolation which is free from perturbative singularities and can be used to generate meaningful pre-showered events. When generating LHEs according to the POWHEG formula, we can generate events using the smooth isolation prescription of the photons according to the formula (Frixione-type isolation with $\epsilon_{\gamma}=n=1$ )

$$
E_{\perp, \text { had }}=\sum_{i \in \text { partons }} E_{\perp, i} \Theta\left(\delta-R\left(p_{\gamma}, p_{i}\right)\right) \leq E_{\perp, \gamma}\left(\frac{1-\cos \delta}{1-\cos \delta_{0}}\right)
$$

for all $\delta \leq \delta_{0}$, where $\delta_{0}$ is a sufficiently small, pre-defined number. This isolation can be considered as a generation isolation, $\Theta_{\text {isol }}^{\text {gen }}\left(\delta_{0}\right)$. Then the inclusive cross section can be decomposed as

$$
\sigma_{\text {incl }}=\sigma_{\text {incl }} \Theta_{\text {isol }}^{\text {gen }}+\sigma_{\text {incl }}\left(1-\Theta_{\text {isol }}^{\text {gen }}\right),
$$

where the first term on the right hand side is the perturbatively computable cross section with smooth photon isolation of eq. (4.2). The second one contains non-perturbative contribution to, therefore, cannot be computed in perturbation theory. Thus eq. (4.3) can be considered as (an unconventional) factorization of the quark-photon singularity into non-perturbative contribution. Applying a physical isolation on eq. (4.3), we obtain the experimentally measurable cross section for isolated photon production,

$$
\sigma_{\text {isol }}^{\text {exp }}=\sigma_{\text {incl }} \Theta_{\text {isol }}^{\text {exp }}=\sigma_{\text {incl }} \Theta_{\text {isol }}^{\text {gen }}\left(\delta_{0}\right) \Theta_{\text {isol }}^{\text {exp }}+\sigma_{\text {incl }}\left(1-\Theta_{\text {isol }}^{\text {gen }}\left(\delta_{0}\right)\right) \Theta_{\text {isol }}^{\exp } .
$$


If the experimental isolation is simply a tighter version of the smooth isolation of eq. (4.2), then the non-perturbative contribution trivially vanishes, as $\left(1-\Theta_{\text {isol }}^{\text {gen }}\left(\delta_{0}\right)\right) \Theta_{\text {isol }}^{\text {exp }}=0$. Thus the events generated with smooth isolation can be used to make such physical prediction. If the physical isolation is the cone-type isolation of eq. (4.1), then the non-perturbative contribution is non-zero. Nevertheless, we shall argue that if the generation isolation is sufficiently loose and the photon is sufficiently hard, then for cone-type isolation with values of parameters used in the experiments, the non-perturbative contribution is negligible within the accuracy of the perturbative one, thus the first term still gives a sufficiently good description of data.

First let us note that left hand side of eq. (4.4) is independent of $\delta_{0}$, so must be the right hand side, too. Below we shall demonstrate that for sufficiently loose generation isolation, in the range $\delta_{0}^{\text {gen }} \in[0.01,0.1]$, the term $\sigma_{\text {incl }} \Theta_{\text {isol }}^{\text {gen }} \Theta_{\text {isol }}^{\text {exp }}$ obtained with usual experimental cone-type isolation of eq. (4.1), depends on $\delta_{0}^{\text {gen }}$ very little. As a result, the second term on the right hand side of eq. (4.4) has to be almost independent of $\delta_{0}^{\text {gen }}$, too. Although this second term is not computable in perturbation theory, making $\Theta_{\text {isol }}^{\text {gen }}$ looser, it decreases, and we expect it becomes negligible within the accuracy of the calculation, when $\delta_{0}^{\text {gen }} \leq 0.05$ and the photon is hard. We shall discuss the accuracy of this assumption further in section 6 by comparing the prediction from the first term on the right hand side of eq. (4.4) to the experimentally measured cross section for a specific process. This means that the first term, $\sigma_{\text {incl }} \Theta_{\text {isol }}^{\text {gen }} \Theta_{\text {isol }}^{\text {exp }}$ approximates the experimentally isolated hard photon cross section within the accuracy of the prediction.

With such a generation isolation we can generate a sufficiently inclusive sample of preshowered events. On the events prepared this way it is easy to apply a close-to-experiment type of cut such as eq. (4.1), the quark-photon singularity is appropriately screened hence allowing for a small hadronic (or partonic) activity in the cone around the photon and cannot lead to infinite predictions. This procedure of making theoretical predictions is made possible by the generation of LHEs as opposed to producing differential distributions directly, as in the case of computing cross sections at fixed order in perturbation theory beyond LO accuracy.

\section{Independence of the generation isolation}

We generate an almost inclusive event sample with a loose photon isolation. The generation isolation parameter $\delta_{0}^{\text {gen }}$ should be chosen such that the distributions with experimental photon isolation obtained at various stages of event simulation (from LHEs, after parton shower and after full SMC) should be independent of it. In order to see this independence, we generated events with three different generation isolation values: $\delta_{0}^{\text {gen }} \in\{0.01,0.05,0.1\}$. These event generations are done with parameters listed in section 3 . Then we compare the predictions made with different values of $\delta_{0}^{\text {gen }}$ at various stages of the event simulation. Although the particle content can be different at different stages of event evolution, we kept the set of cuts applied to the events the same:

- There is a cut on the transverse momentum of the hardest photon: $p_{\perp, \gamma}>30 \mathrm{GeV}$.

- The hardest photon should be central: $\left|y_{\gamma}\right|<2.5$. 

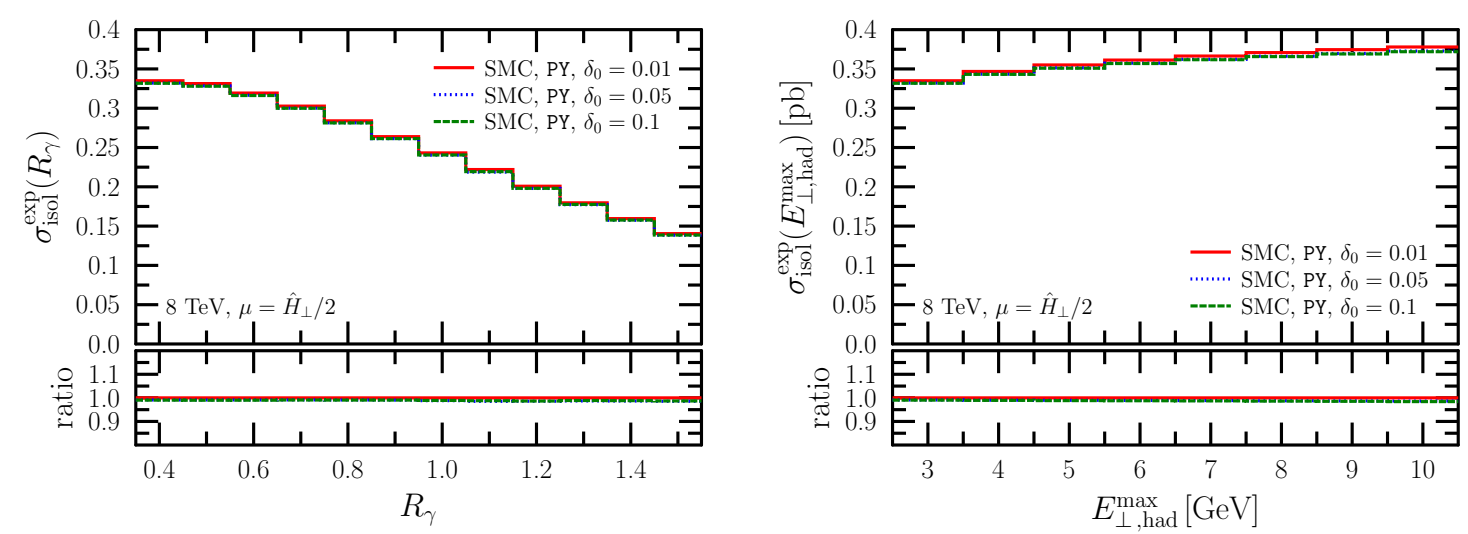

Figure 6. Isolated photon cross sections obtained after full SMC with different generation isolations using cuts listed in the text, as a function of a) the radius of experimental photon isolation cone, b) the hadronic leakage inside the photon isolation cone.

- A jet algorithm is applied using the anti- $k_{\perp}$ algorithm [25] provided by FastJet [26, 27] with $p_{\perp}^{j}>30 \mathrm{GeV}$ and $R=0.4$.

- The hardest photon should be well-isolated from the jets: $\Delta R(\gamma, j)>0.4$ measured on the rapidity-azimuthal angle plane.

- A hadronic (or partonic) leakage is allowed in an $R_{\gamma}=0.4$ cone around the photon according to eq. (4.1) with $E_{\perp, \text { had }}^{\max }=3 \mathrm{GeV}$.

We have checked that for $\delta_{0}^{\text {gen }} \in[0.01,0.1]$ the physical predictions depend marginally on $\delta_{0}^{\text {gen }}$ at all stages of the event evolution, but show here only for predictions obtained at the hadronic stage, i.e. after SMC. The cross section values after full SMC and given selection cuts are presented as a function of the radius of experimental photon isolation cone, and of the hadronic leakage inside the photon isolation cone in figure 6 . We see that independently of these parameters (within the ranges shown here), the physical cross section depends on the generation isolation weakly.

For kinematic distributions we find even smaller dependence on $\delta_{0}^{\text {gen }}$. Six sample distributions are presented on figures 7-9.

\section{Estimation of the non-pertrubative contribution}

In eq. (4.4) we decomposed the isolated photon cross section into a perturbatively computable part (first term) and a non-perturbative contribution (second term). Furthermore, we argued that provided $\delta_{0}^{\text {gen }}$ sufficiently small, we expect the non-perturbative contribution to be small. This statement can only be verified by explicit comparison to experimental data, which is presently not possible for isolated photon production in association with a $t \bar{t}$ pair. It is possible however, for the case of massive vector boson + isolated photon production for which the ATLAS collaboration published results for both isolated photon +0 jet (exclusive) and isolated photon $+N(\geq 0)$ jets (inclusive) in the final state [28]. This final state has also been considered recently at NLO accuracy interfaced to a shower 

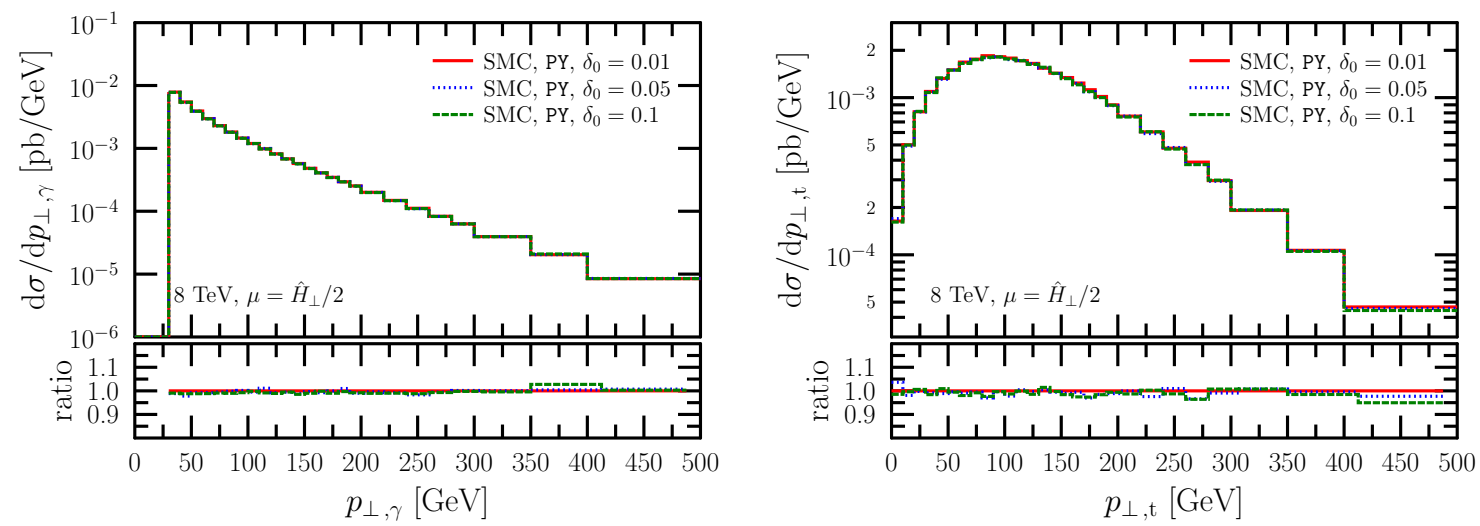

Figure 7. Transverse-momentum distribution for the hardest photon and the t-quark after parton shower and hadronization with PYTHIA for smooth generation isolation with $\delta_{0}^{\text {gen }} \in[0.01,0.1]$. On the lower panels the ratios of the predictions are shown.
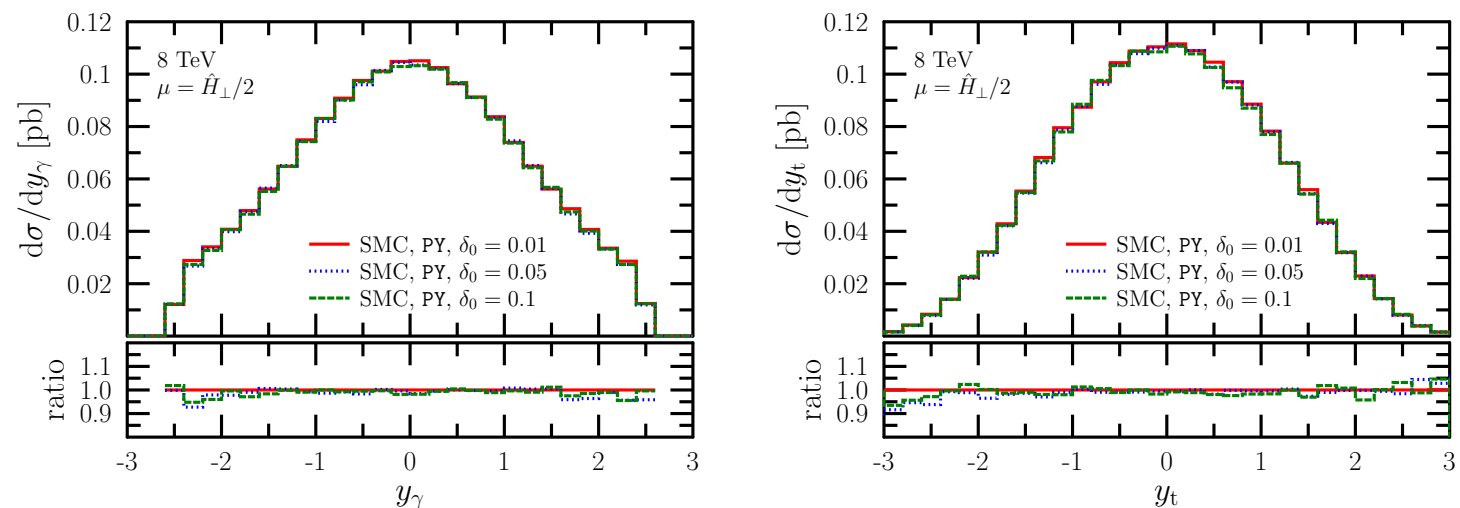

Figure 8. The same as figure 7 but for the rapidities of the hardest-photon and the t-quark.
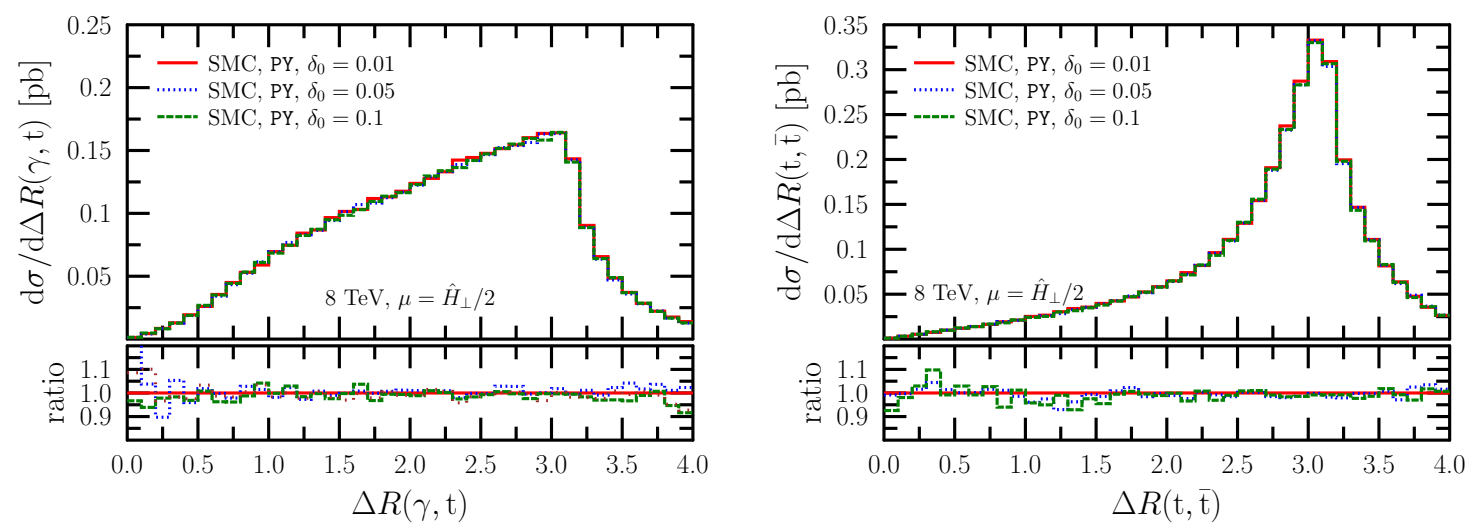

Figure 9. The same as figure 7 but for separations in the rapidity-azimuthal angle plane.

generator according to the POWHEG prescription supplemented with the MiNLO procedure [29]. In this work the fixed order result is matched to an interleaved QCD+QED parton shower, in such a way that the contribution arising from hadron fragmentation into photons is fully modeled. Thus for this process the comparison is possible not only 

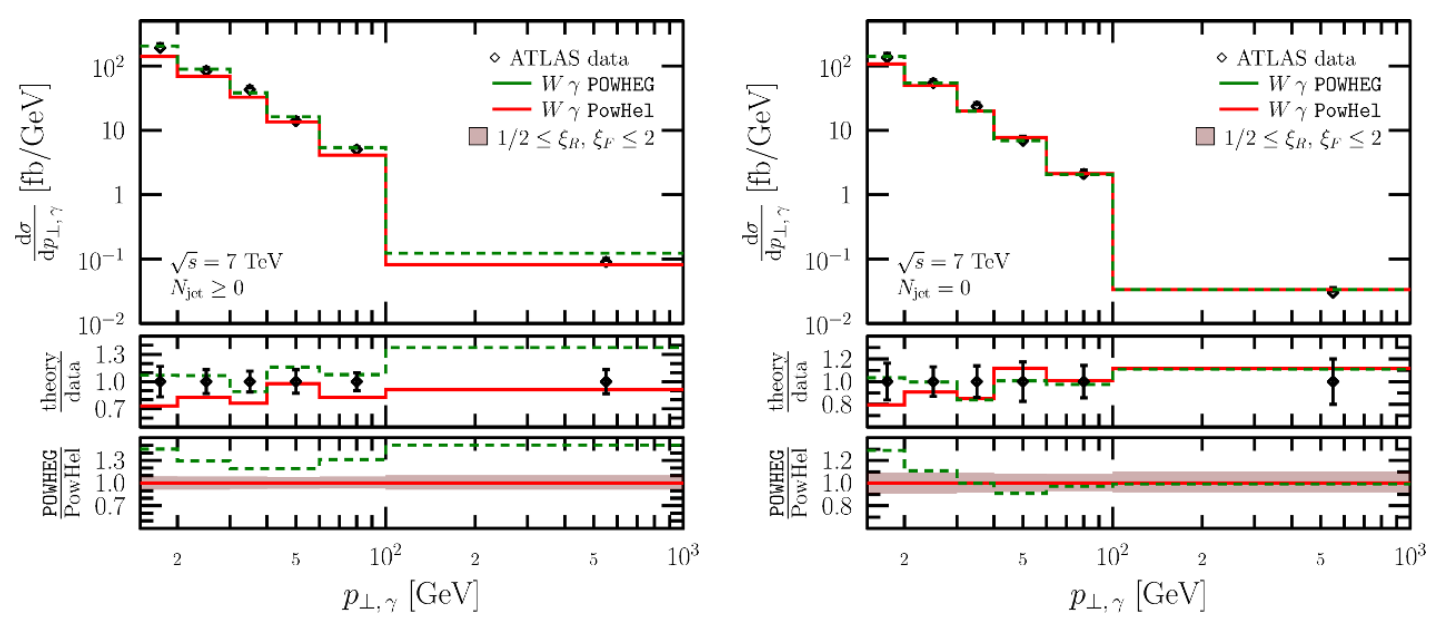

Figure 10. Transverse-momentum distribution of the isolated photon for the a) $W+\gamma+N \geq 0$ jets and b) $W+\gamma+0$ jet final states. The lower panels show the ratio of the predictions to the data.

for experimental results, but also with a theoretical prediction where the fragmentation is included through a shower model.

Within PowHel the $W \gamma$ process can be implemented straightforwardly. We generated events for $W \gamma$ production with the same three values of $\delta_{0}^{\text {gen }}$ as in the case of $\mathrm{t} \overline{\mathrm{t}} \gamma$ production and checked that the predictions from the pre-showered events agree with those at NLO accuracy, just as in the case of $t \overline{\mathrm{t}} \gamma$ production in section 3. Next, we checked the dependence on the generation isolation parameter, similarly as in section 5 and found that the perturbative prediction depends weakly (below $10 \%$ for the exclusive and below $5 \%$ for the inclusive case) on the choice of $\delta_{0}^{\text {gen }}$ in the range $\delta_{0}^{\text {gen }} \in[0.01,0.1]$ if a similar physical isolation is used as in section 5 . Thus we have implemented the event selection of ref. [28] and made predictions for the inclusive case and for the exclusive case using events obtained with $\delta_{0}^{\text {gen }}=0.05$.

We show our predictions for the transverse-momentum distribution of the isolated photon, compared to the predictions of ref. [29] and the results of ref. [28] in figure 10.

In general, we find that our approach gives a good description of the data if the radiated photon is harder than the accompanying jets. Thus for the exclusive case, the data overlap with our predictions for the full range within the uncertainty of the prediction. In fact, for this case the two theoretical predictions also coincide within the scale dependence band except for the first bin. For the inclusive case the two predictions differ and the difference, in principle, may be attributed to the neglected fragmentation contribution. However, it should be noted that the predictions of ref. [29] do not use non-perturbative information extracted from data, but a model of fragmentation. At the present accuracy of the data it is difficult to make a clear conclusion which prediction is favoured by experiment, but the following general trend seems to emerge: the harder the photon the better the agreement between our prediction and the data, while for the case of matched NLO to an interleaved QCD+QED parton shower, the agreement is better for small transverse momenta, with transition around $60 \mathrm{GeV}$ for the given selection cuts. 

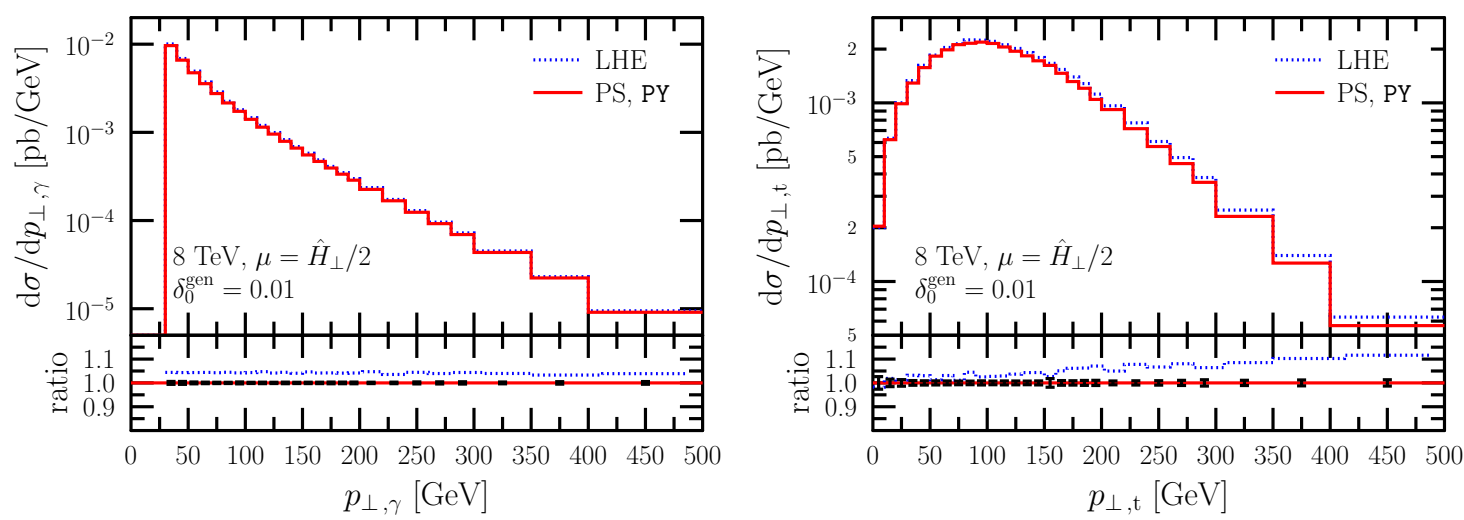

Figure 11. Transverse-momentum distribution for the photon and t-quark at the LHE stage and after parton shower. The lower panel shows the LHE/PS ratio.

\section{$7 \quad$ Effect of the parton shower}

In the previous sections we estimated the effect of the neglected non-perturbative contribution, as well as demonstrated that predictions for isolated photon cross section made with full SMC do not depend upon the sufficiently small generation-isolation. In fact, we also checked that the latter is true at various stages of event simulation (LHE, PS and SMC). To quantify the effect of the parton shower and in the next section to present physical predictions after full SMC we decided to use $\delta_{0}^{\text {gen }}=0.01$ in our generation isolation. For this comparison we used the setup of section 5. Our standard distributions can be found on figures 11-13. While for rapidities and separations the difference between the LHE and PS stages only manifest in an overall change in normalization, for the transverse-momentum distribution of the t-quarks the change is not only a constant factor in normalization, but there is even a change in the shape. As we expect, the shower softens the spectra. This softening added to the difference between the predictions of LHEs and at NLO suggests very small PS effect at high transverse momenta. (We cannot compare figures 3 and 11 directly as photon isolations are different.) In the case of the photon $p_{\perp}$ the change remains small, around $5 \%$, while for the transverse momentum of the t-quark it reaches even $12 \%$ when the $p_{\perp}$ approaches $500 \mathrm{GeV}$. If our default, rather tight, criterion on the allowed hadronic leakage is loosen up (going from $3 \mathrm{GeV}$ to $10 \mathrm{GeV}$ ) the difference observed in the photon transverse-momentum distribution remains more-or-less the same, but in the case of the transverse momentum of the t-quark the difference drops below $10 \%$ in the high- $p_{\perp}$ region. The relaxation in the hadronic leakage condition results in a smaller difference, $\sim 1 \%$, for rapidities and separations.

\section{Predictions}

We conclude with a simple phenomenological study at the hadron level. To this end PYTHIA-6.4.25 was chosen to decay, shower and finally hadronize the events. The event sample with $\delta_{0}^{\text {gen }}=0.01$ at $8 \mathrm{TeV}$ was selected, PYTHIA was run with the 2010 Perugia 

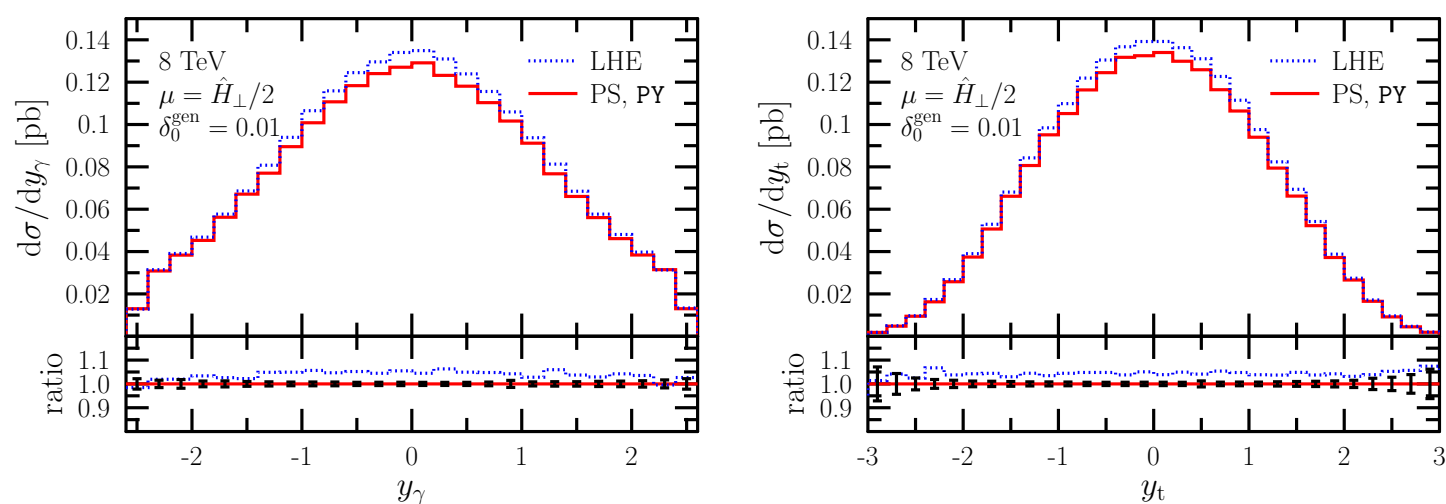

Figure 12. The same as figure 11 but for the rapidities of the photon and t-quark.
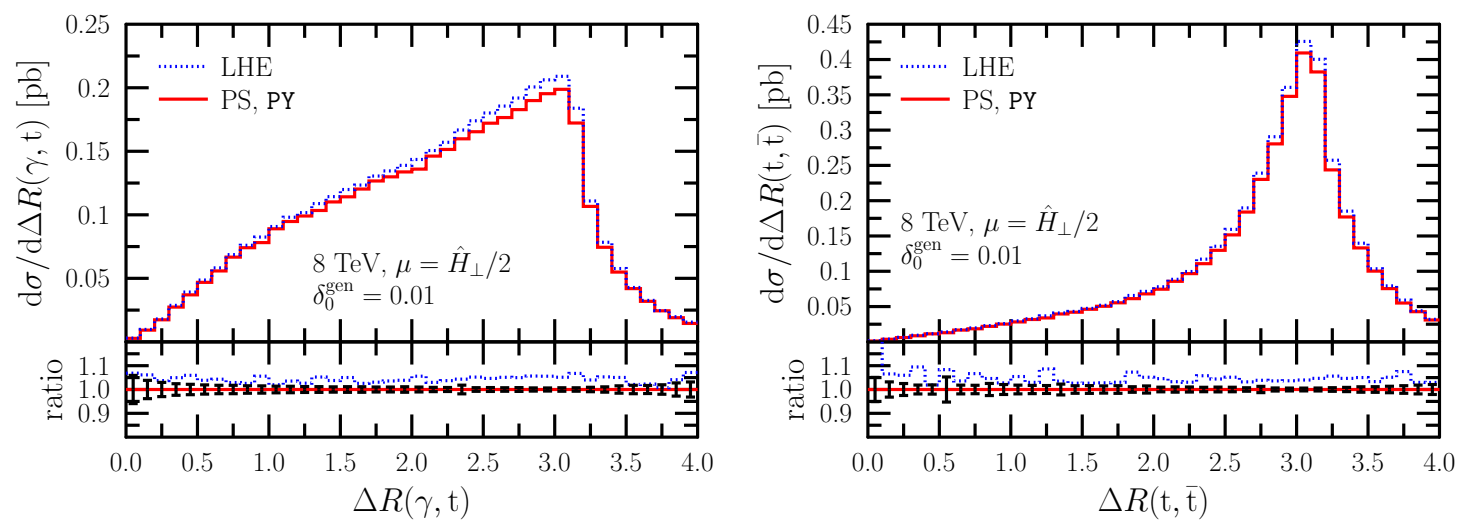

Figure 13. The same as figure 11 but for the separation of the photon-t and $t \bar{t}$ systems.

tune [30], omitting photon showers, making $\tau^{ \pm}$and $\pi^{0}$ stable and we turned off multiparticle interactions. The cuts employed in this analysis were the following:

- The analysis was done in the semileptonic decay-channel by requesting exactly one hard lepton or antilepton in the final state with $p_{\perp, \ell}>30 \mathrm{GeV}$, the (anti)lepton had to be isolated from all the jets with $\Delta R(\ell, j)>0.4$.

- The final state had to contain one hard photon in the central region, $\left|y_{\gamma}\right|<2.5$ with $p_{\perp, \gamma}>30 \mathrm{GeV}$, isolated from all the jets by $\Delta R(\gamma, j)>0.4$. A minimal hadronic leakage was allowed in a $R_{\gamma}=0.4$ cone around the photon with $E_{\perp \text {,had }}^{\max }=3 \mathrm{GeV}$ according to eq. (4.1).

- The (anti)lepton and photon had to be separated from each other, $\Delta R(\gamma, \ell)>0.4$.

- Jets were reconstructed with the anti- $k_{\perp}$ algorithm [25] with $R=0.4$ and $p_{\perp}^{j}>$ $30 \mathrm{GeV}$.

- The event had to have significant missing transverse momentum, $\not p_{\perp}>30 \mathrm{GeV}$.

In our calculation, throughout, a different scale choice was used than that in the literature [20] for $\mathrm{t} \overline{\mathrm{t}} \gamma$ production. Our default scale choice, the half the sum of transverse 

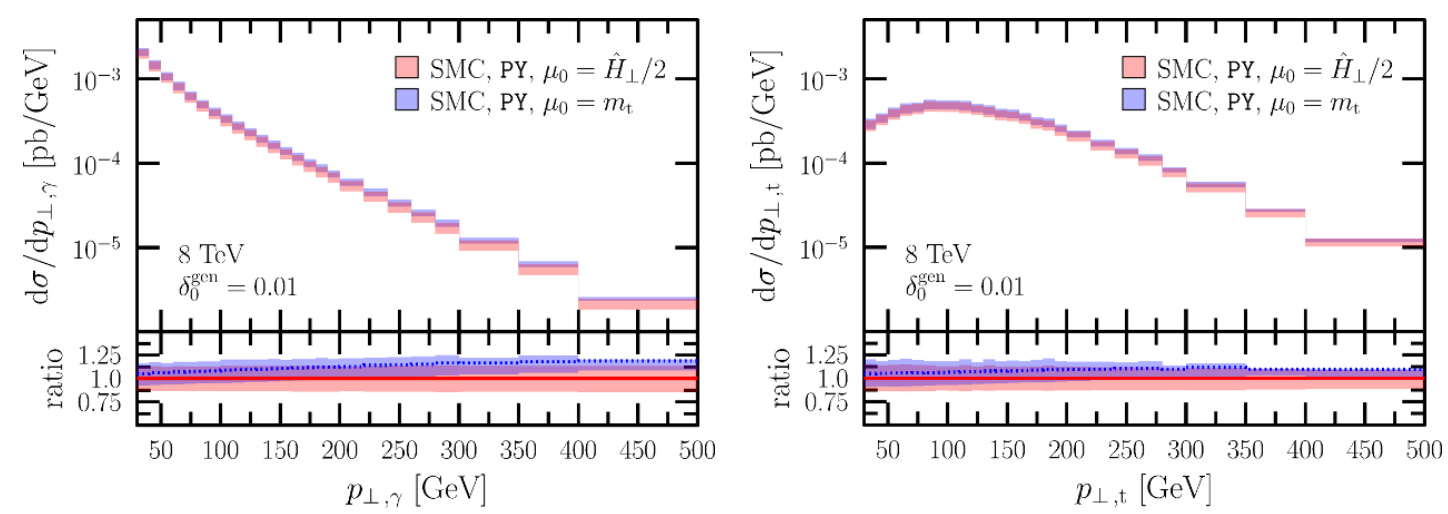

Figure 14. Transverse momentum distribution for the photon and the t-quark at the hadronic stage. On the lower panel the ratio of predictions to that obtained with our default scale choice is shown.

masses $\hat{H}_{\perp} / 2$ was already motivated in [31]. To see the difference between the two scale choices a scale-uncertainty study is performed and scale-uncertainty bands are shown for the distributions obtained at the hadron level. The renormalization and factorization scales are defined as $\mu_{R}=\xi_{R} \mu_{0}$ and $\mu_{F}=\xi_{F} \mu_{0}$, respectively, and the band is formed as the upperand lower-bounding envelopes of distributions taken with

$$
\left(\xi_{R}, \xi_{F}\right) \in\left\{\left(\frac{1}{2}, \frac{1}{2}\right),\left(\frac{1}{2}, 1\right),\left(1, \frac{1}{2}\right),(1,1),(1,2),(2,1),(2,2)\right\} .
$$

The antipodal choices $((1 / 2,2)$ and $(2,1 / 2))$ are left out. When these are included, the uncertainty band for rapidities and separations are unchanged while for transverse momenta in the large transverse-momentum region the band widens by a few percent.

In figure 14 the transverse momenta of the photon and the t-quark are shown. The momentum of the t-quark is reconstructed just like in the previous cases using MCTRUTH. Taking a look at the transverse momentum of the photon the static scale results in a narrower band with a shrinking width. This hints a cross-over point at a higher $p_{\perp}$ value, while in the case of the dynamical scale the band, although wider, keeps the same width all across the whole plotted transverse-momentum spectrum. While for the $p_{\perp}$-distribution of the photon the presence of a cross-over point is only hinted by the narrowing uncertainty band, for the transverse momentum of the t-quark it is indeed visible around $350 \mathrm{GeV}$. Until this point the uncertainty band taken with the static scale decreases in width than after opens up. This is somehow expected since a highly boosted t-quark with a heavy companion anti-t and a photon correspond to a system with a large summed transverse mass hence lying far away from the central scale $m_{\mathrm{t}}$.

In figure 15 the spectrum of the transverse momentum of the charged lepton and that of the missing momentum are shown. For both distributions a cross-over can be seen around $250 \mathrm{GeV}$ when static scale is used. The dynamical scale choice appears to give reliable scale dependence over the whole plotted range for these observables.

If we turn our attention to the separations between the photon and the t-quark, as well as between the photon and the charged lepton, measured in the rapidity-azimuthal 

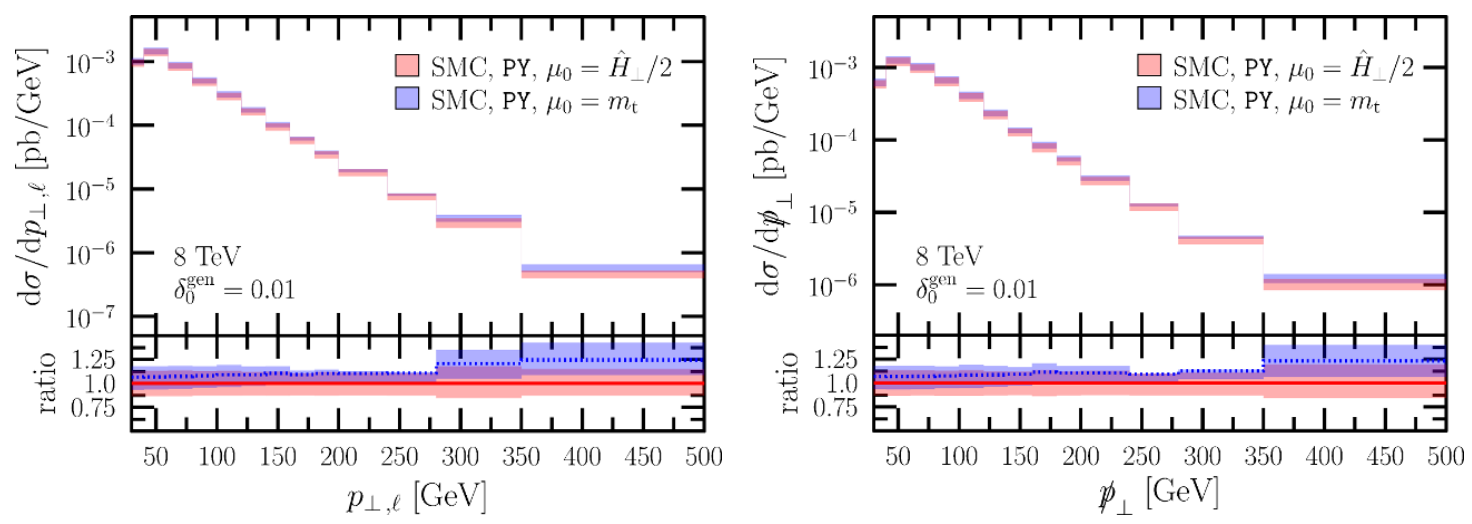

Figure 15. The same as figure 14 but for the spectra of the transverse momentum of the charged (anti)lepton and the missing momentum.
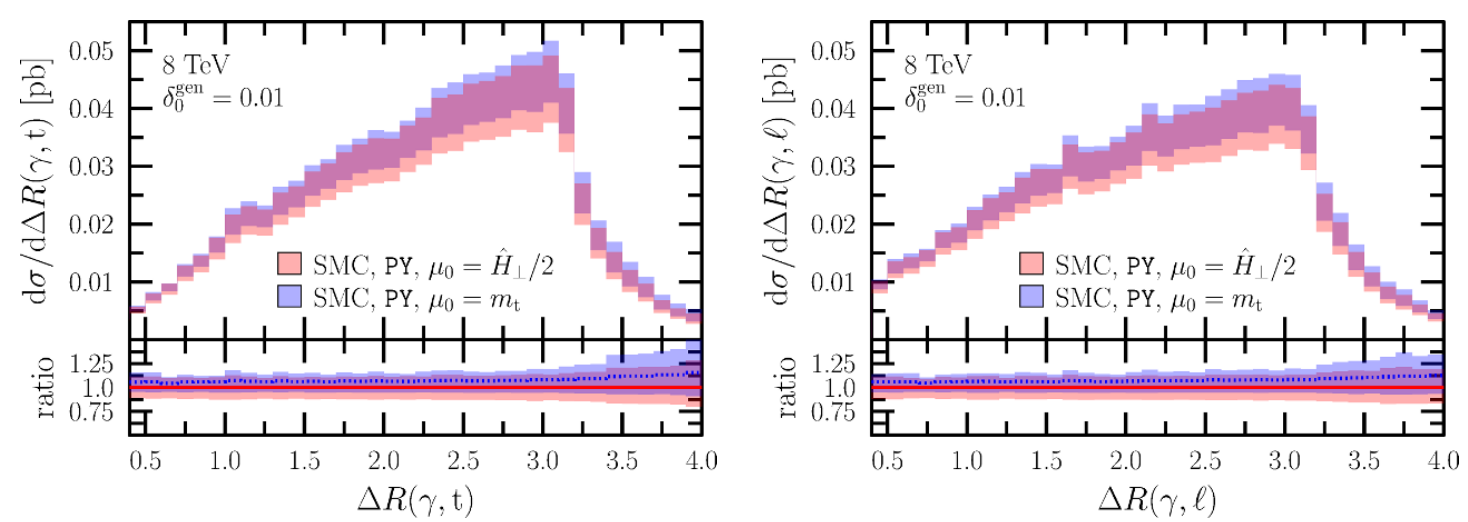

Figure 16. The same as figure 14 but for separations measured in the rapidity-azimuthal angle plane.

angle plane, we do not find significant difference between the two scale choices, as seen in figure 16. The static scale gives somewhat higher cross section and a slightly narrower uncertainty band below $\Delta R=\pi$ and larger scale dependence above. Similar conclusions can be drawn from the rapidity distributions for the photon and the (anti)lepton shown in figure 17. In general, the scale dependence is moderate, below $20 \%$ for both scale choices and all observables, except for the predictions at large transverse momenta with the static scale.

\section{Conclusions}

In this paper we presented a new way to make predictions for the hadroproduction of isolated photons which uses event samples that emerge in simulations aimed at matching predictions at NLO accuracy with PS. Our approach uses only the direct-photon contribution, i.e. we neglect the fragmentation. We demonstrated that the presence of a sufficiently small smooth isolation of the direct photons, applied during generation of the events, does not affect the physical predictions, within the numerical accuracy of the calculation. Hence it can be used to generate sufficiently inclusive pre-showered event samples. The 

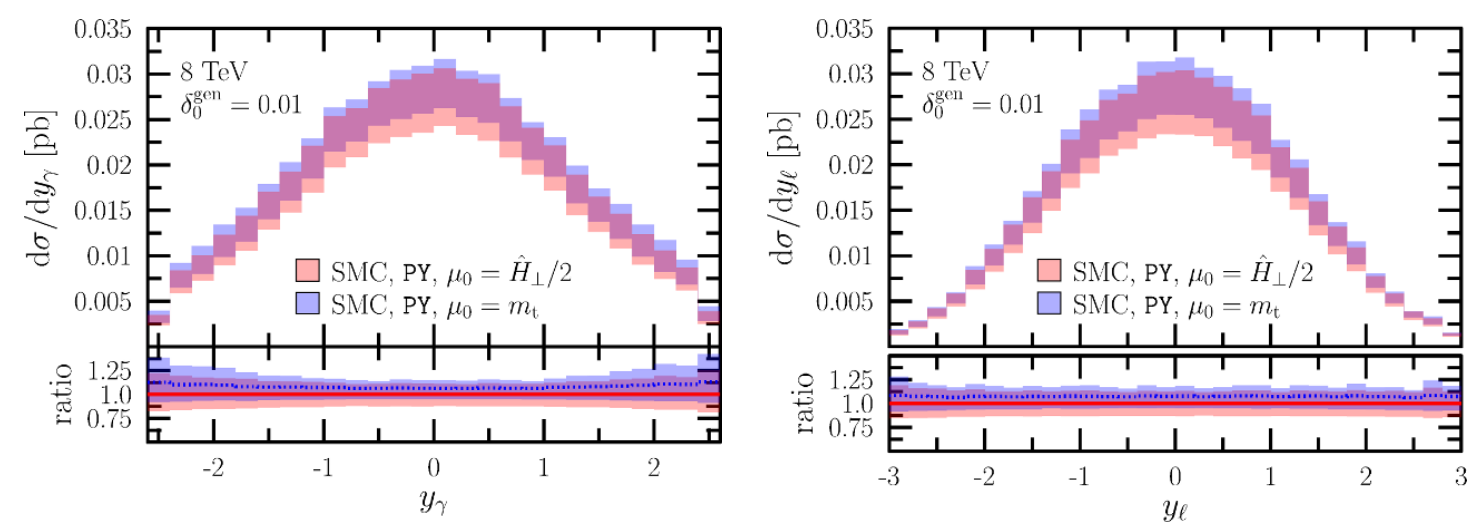

Figure 17. The same as figure 14 but for the rapidity distributions of the photon and (anti)lepton.

pre-showered events obtained this way can be further showered and hadronized to obtain differential distributions at the hadronic stage, which include NLO QCD corrections in the hard process, and either smooth or standard experimental photon isolation can be applied.

Using the POWHEG method one can make predictions at various stages of the event simulation. In particular, for most of the phenomenologically interesting distributions we estimate fairly small (about $10 \%$, or less) corrections for the $t \bar{t} \gamma$ final state due to the parton shower. We also studied the dependence of our predictions on the renormalization and factorization scales and found small and rather uniform scale dependence for the default scale $\hat{H}_{\perp} / 2$.

The events generated with a loose generation isolation contain only perturbative information, we neglected the non-perturbative (fragmentation) contribution. We argued that making the generation isolation looser, the fragmentation contribution should decrease. Therefore in case of sufficiently loose generation isolation the fragmentation can be neglected within the expected uncertainty of matched NLO+PS predictions if the photon is harder than the accompanying jets. This statement is trivially true if the experimental isolation is a tighter version of the smooth isolation than that employed for event generation. We demonstrated the fragmentation also becomes negligible in the case of hadroproduction of a $W$ boson in association with a hard isolated photon by comparing our predictions to measured data if the photon is harder than the accompanying jets. Our method is completely general and can be used to any process with isolated hard photons in the final state, in particular also for $\mathrm{t} \overline{\mathrm{t}}$ production in association with hard isolated photons.

\section{Acknowledgments}

This research was supported by the Hungarian Scientific Research Fund grant K-101482, the European Union and the European Social Fund through Supercomputer, the national virtual lab TAMOP-4.2.2.C-11/1/KONV-2012-0010 and the LHCPhenoNet network PITNGA-2010-264564 projects.

Open Access. This article is distributed under the terms of the Creative Commons Attribution License (CC-BY 4.0), which permits any use, distribution and reproduction in any medium, provided the original author(s) and source are credited. 


\section{References}

[1] ATLAS collaboration, Observation of a new particle in the search for the standard model Higgs boson with the ATLAS detector at the LHC, Phys. Lett. B 716 (2012) 1 [arXiv:1207.7214] [INSPIRE].

[2] CMS collaboration, Observation of a new boson at a mass of $125 \mathrm{GeV}$ with the CMS experiment at the LHC, Phys. Lett. B 716 (2012) 30 [arXiv:1207.7235] [InSPIRE].

[3] T. Kinoshita, Mass singularities of Feynman amplitudes, J. Math. Phys. 3 (1962) 650 [INSPIRE].

[4] T. Lee and M. Nauenberg, Degenerate systems and mass singularities, Phys. Rev. 133 (1964) B1549.

[5] S. Catani, S. Dittmaier, M.H. Seymour and Z. Trócsányi, The dipole formalism for next-to-leading order QCD calculations with massive partons, Nucl. Phys. B 627 (2002) 189 [hep-ph/0201036] [INSPIRE].

[6] Z. Kunszt and Z. Trócsányi, QCD corrections to photon production in association with hadrons in $e^{+} e^{-}$annihilation, Nucl. Phys. B 394 (1993) 139 [hep-ph/9207232] [InSPIRE].

[7] S. Frixione, Isolated photons in perturbative QCD, Phys. Lett. B 429 (1998) 369 [hep-ph/9801442] [INSPIRE].

[8] S. Catani, M. Fontannaz and E. Pilon, Factorization and soft gluon divergences in isolated photon cross-sections, Phys. Rev. D 58 (1998) 094025 [hep-ph/9803475] [INSPIRE].

[9] S. Frixione and B.R. Webber, Matching NLO QCD computations and parton shower simulations, JHEP 06 (2002) 029 [hep-ph/0204244] [INSPIRE].

[10] P. Nason, A new method for combining NLO QCD with shower Monte Carlo algorithms, JHEP 11 (2004) 040 [hep-ph/0409146] [INSPIRE].

[11] S. Frixione, P. Nason and C. Oleari, Matching NLO QCD computations with parton shower simulations: the POWHEG method, JHEP 11 (2007) 070 [arXiv:0709.2092] [INSPIRE].

[12] J. Alwall et al., The automated computation of tree-level and next-to-leading order differential cross sections and their matching to parton shower simulations, JHEP 07 (2014) 079 [arXiv: 1405.0301] [INSPIRE].

[13] S. Alioli, P. Nason, C. Oleari and E. Re, A general framework for implementing NLO calculations in shower Monte Carlo programs: the POWHEG BOX, JHEP 06 (2010) 043 [arXiv:1002.2581] [INSPIRE].

[14] G. Bevilacqua et al., HELAC-NLO, Comput. Phys. Commun. 184 (2013) 986 [arXiv:1110.1499] [INSPIRE].

[15] M.V. Garzelli, A. Kardos, C.G. Papadopoulos and Z. Trócsányi, Standard model Higgs boson production in association with a top anti-top pair at NLO with parton showering, Europhys. Lett. 96 (2011) 11001 [arXiv:1108.0387] [INSPIRE].

[16] LHC Higgs Cross Section Working Group collaboration, S. Dittmaier et al., Handbook of LHC Higgs Cross Sections: 1. Inclusive observables, arXiv:1101.0593 [INSPIRE].

[17] M.V. Garzelli, A. Kardos, C.G. Papadopoulos and Z. Trócsányi, $t \bar{t} W^{+-}$and $t \bar{t} Z$ hadroproduction at NLO accuracy in QCD with parton shower and hadronization effects, JHEP 11 (2012) 056 [arXiv:1208.2665] [INSPIRE]. 
[18] A. Kardos, C. Papadopoulos and Z. Trócsányi, Top quark pair production in association with a jet with NLO parton showering, Phys. Lett. B 705 (2011) 76 [arXiv:1101.2672] [INSPIRE].

[19] J. Alwall et al., A standard format for Les Houches event files, Comput. Phys. Commun. 176 (2007) 300 [hep-ph/0609017] [INSPIRE].

[20] K. Melnikov, M. Schulze and A. Scharf, QCD corrections to top quark pair production in association with a photon at hadron colliders, Phys. Rev. D 83 (2011) 074013 [arXiv: 1102.1967] [INSPIRE].

[21] S. Alioli, P. Nason, C. Oleari and E. Re, Vector boson plus one jet production in POWHEG, JHEP 01 (2011) 095 [arXiv: 1009.5594] [InSPIRE].

[22] A. Kardos, P. Nason and C. Oleari, Three-jet production in POWHEG, JHEP 04 (2014) 043 [arXiv: 1402.4001] [INSPIRE].

[23] S. Alioli, P. Nason, C. Oleari and E. Re, NLO Higgs boson production via gluon fusion matched with shower in POWHEG, JHEP 04 (2009) 002 [arXiv: 0812.0578] [INSPIRE].

[24] M.R. Whalley, D. Bourilkov and R.C. Group, The Les Houches accord PDFs (LHAPDF) and LHAGLUE, hep-ph/0508110 [INSPIRE].

[25] M. Cacciari, G.P. Salam and G. Soyez, The anti- $k_{t}$ jet clustering algorithm, JHEP 04 (2008) 063 [arXiv: 0802.1189] [INSPIRE].

[26] M. Cacciari, G.P. Salam and G. Soyez, FastJet user manual, Eur. Phys. J. C 72 (2012) 1896 [arXiv: 1111.6097] [INSPIRE].

[27] M. Cacciari and G.P. Salam, Dispelling the $N^{3}$ myth for the $k_{t}$ jet-finder, Phys. Lett. B 641 (2006) 57 [hep-ph/0512210] [INSPIRE].

[28] ATLAS collaboration, Measurements of $W \gamma$ and $Z_{\gamma}$ production in pp collisions at $\sqrt{s}=7 \mathrm{TeV}$ with the ATLAS detector at the LHC, Phys. Rev. D 87 (2013) 112003 [arXiv: 1302.1283] [INSPIRE].

[29] L. Barze et al., Wr production in hadronic collisions using the POWHEG+MiNLO method, JHEP 12 (2014) 039 [arXiv: 1408.5766] [INSPIRE].

[30] P.Z. Skands, Tuning Monte Carlo generators: the Perugia tunes, Phys. Rev. D 82 (2010) 074018 [arXiv: 1005 . 3457] [INSPIRE].

[31] A. Kardos and Z. Trócsányi, Hadroproduction of $t \bar{t}$ pair with a b $\bar{b}$ pair using PowHel, J. Phys. G 41 (2014) 075005 [arXiv:1303.6291] [InSPIRE]. 